\title{
Towards an Algebraic Network Information Theory: Distributed Lossy Computation of Linear Functions
}

\author{
Sung Hoon Lim \\ KIOST \\ shlim@kiost.ac.kr
}

\author{
Chen Feng \\ University of British Columbia \\ chen.feng@ubc.ca
}

\author{
Adriano Pastore
}

CTTC/CERCA

adriano.pastore@cttc.cat

\author{
Bobak Nazer \\ Boston University \\ bobak@bu.edu
}

\author{
Michael Gastpar \\ EPFL
}

michael.gastpar@epfl.ch

\begin{abstract}
Consider the important special case of the $K$-user distributed source coding problem where the decoder only wishes to recover one or more linear combinations of the sources. The work of Körner and Marton demonstrated that, in some cases, the optimal rate region is attained by random linear codes, and strictly improves upon the best-known achievable rate region established via random i.i.d. codes. Recent efforts have sought to develop a framework for characterizing the achievable rate region for nested linear codes via joint typicality encoding and decoding. Here, we make further progress along this direction by proposing an achievable rate region for simultaneous joint typicality decoding of nested linear codes. Our approach generalizes the results of Körner and Marton to computing an arbitrary number of linear combinations and to the lossy computation setting.
\end{abstract}

Index Terms-Nested linear codes; joint decoding; distributed source-coding; function computation

\section{INTRODUCTION}

Consider the $K$-user distributed lossy computation system in Figure 1. Let $\left(X_{1}, \ldots, X_{K}, Y\right) \sim p\left(x_{1}, \ldots, x_{K}, y\right)$ be a $K+1$ discrete memoryless source (DMS) where $X_{k}$, $k \in \mathcal{K}=\{1, \ldots, K\}$ and $Y$ take values in a finite field $\mathbb{F}_{\mathrm{q}}$ of order q. ${ }^{1}$ For some matrix $\mathrm{A} \in \mathbb{F}_{\mathrm{q}}^{J \times K}, J \leq K$, let $W_{\mathrm{A}}=\mathrm{A}\left[X_{1}, \ldots, X_{K}\right]^{\mathrm{T}}$, denote the desired linear combinations where $(\cdot)^{\top}$ is the transpose operator. User $k \in \mathcal{K}$ observes a discrete memoryless source $X_{k}$, and the decoder with side information $Y$ wishes to recover these $J$ linear combinations of the sources up to some distortion as measured by the functions $d_{j}\left(w_{\mathrm{A}_{j}}, \hat{w}_{\mathrm{A}_{j}}\right), j \in[1: J]$, where $\mathrm{A}_{j}$ is the $j$-th row of $A$ and $\hat{w}_{A_{j}}$ is our estimate of the corresponding linear combination $w_{A_{j}}$.

For the special case of $K=2, J=1, \mathrm{~A}=[1,1], Y=\emptyset$, lossless, and binary symmetric sources, the pioneering work of Körner and Marton [1] demonstrated that random linear codes are optimal and significantly outperform random i.i.d. binning strategies. More recently, several groups have studied the advantages of linear and lattice coding strategies for network source and channel coding. Owing to space limitations, we

S. H. Lim was supported by the National Research Foundation of Korea (NRF) under Grant NRF-2017R1C1B1004192. C. Feng was supported by NSERC Discovery Grant RGPIN-2016-05310. A. Pastore was supported by the Catalan Government under grant 2017 SGR 1479. B. Nazer was supported by NSF grant CCF-1618800. M. Gastpar was supported by the European ERC Starting Grant 259530-ComCom.

${ }^{1}$ We can use a finite field as the underlying alphabet without loss of generality, since other alphabets can be emulated by setting the probabilities of unneeded symbols to zero. only highlight a few papers from the literature on distributed source coding (for discrete memoryless sources) below.

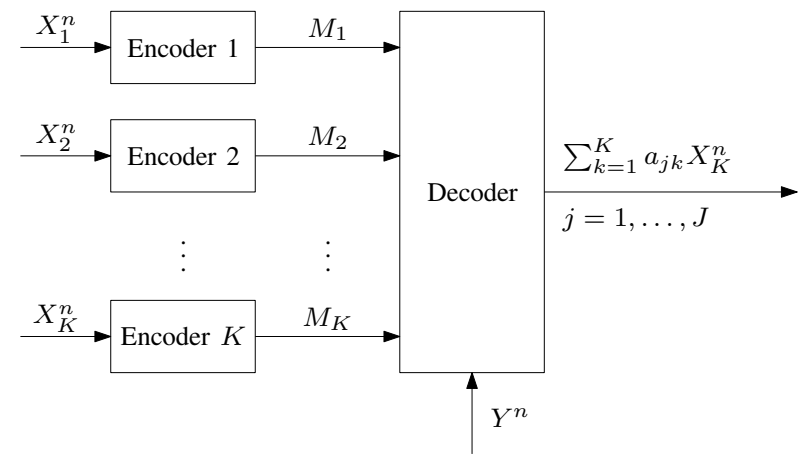

Fig. 1. Distributed source coding for computing multiple linear combinations.

Note that the Slepian-Wolf problem [2] corresponds to the lossless setting with $\mathrm{A}=\mathbf{I}$ (where $\mathbf{I}$ is the identity matrix) and $Y=\emptyset$. It is well-known that the corner points of the SlepianWolf rate region can be attained via nested linear codes coupled with sequential decoders [3], [4]. For the lossless setting with $K=2$ users, $J=1$ linear combination, and $Y=\emptyset$, Han and Kobayashi [5] found necessary and sufficient conditions for the rate region to coincide with the SlepianWolf rate region. Krithivasan and Pradhan proposed a general framework based on Abelian group codes for the special case of $K=2$ users and $J=1$ linear combinations [6]. Subsequently, for general $K$ and $J$ and lossless recovery, Lalitha et al. [7] proposed strategies based on nested linear codes and demonstrated sum-rate optimality for some special cases.

Recent efforts, starting with the work of Padakandla and Pradhan [8]-[10], have sought to unify these compelling examples under an algebraic network information theory [11]-[14]. That is, state-of-the-art achievability arguments for random i.i.d. codes draw upon powerful technical lemmas based on joint typicality encoding and decoding [15], and a similar toolbox is needed to characterize the performance limits of random linear codes. In prior work on the compute-forward problem [11], [16], we derived an achievable rate region for nested linear codes coupled with simultaneous joint typicality decoding. In this paper, we present a complementary analysis: an achievable rate region simultaneous decoding for nested linear codes for distributed source coding of linear functions. 
We closely follow the notation in [15] as well as the definition of typical sets. Two notable departures from this notation are that we denote the set of random variables, $X_{k}$, $k \in \mathcal{S}$ by $X_{\mathcal{S}}$, and we define sets of message indices starting at zero rather than one, $[n]:=\{0, \ldots, n-1\}$. We reserve $\mathcal{K}=\{1, \ldots, K\}$ to denote the full set of users. We use the notation $\mathbb{F}_{\mathrm{q}}$ to denote the finite field of order $q$. We use the notation $\mathbf{I}_{K}$ for the size- $K$ identity matrix, and will sometimes write $\mathbf{I}$ when the size is clear from context. We denote by $\mathrm{e}_{k} \in \mathbb{F}_{\mathrm{q}}^{K}$ the standard basis (row) vector where the $k$-th element is 1 and the rest of the elements are all zero. For a generic matrix $\mathrm{B}$, we denote $\mathrm{B}(\mathcal{S})$ as a submatrix that is composed of the rows of $\mathrm{B}$ with row indices $k \in \mathcal{S}$. For example, we define the matrix $\mathbf{I}_{K}(\mathcal{S}) \in \mathbb{F}_{\mathrm{q}}^{|\mathcal{S}| \times K}$ as the stack of standard basis vectors $\mathrm{e}_{k}, k \in \mathcal{S}$, i.e., the rows of $\mathbf{I}_{K}(\mathcal{S})$ are $\mathrm{e}_{k}, k \in \mathcal{S}$. We will use $\mathbf{0}$ to denote the all-zeros matrix (whose size will be clear from context). We define an empty matrix as a matrix with zero rows or zero columns (or both). We will assume that an empty matrix is full rank with rank 0 . The product of an empty matrix and another matrix is an empty matrix, e.g., if $A$ is a $0 \times 3$ empty matrix and $B$ is a $3 \times 5$ matrix, then $A B$ is an empty matrix of size $0 \times 5$.

\section{Problem Statement}

We consider a $K$-user distributed lossy computation system with $K+1$ correlated, memoryless sources $\left(X_{1}^{n}, \ldots, X_{K}^{n}, Y^{n}\right) \sim \prod_{i=1}^{n} p_{X_{1}, \ldots, X_{K}, Y}\left(x_{1}, \ldots, x_{K}, y\right)$ and distortion functions $d_{j}(\cdot, \cdot), j \in[1: J]$, where $X_{k}$ is the source at encoder $k$ and $Y$ is the receiver side information. Recall that we assume the source symbols are drawn from a finite field $\mathbb{F}_{\mathrm{q}}$, and wish to recover $J$ linear combinations of the form $W_{\mathrm{A}_{j}, i}=\mathrm{A}_{j}\left[X_{1, i}, \ldots, X_{K, i}\right]^{\top}, i \in[1: n]$ where $\mathrm{A}_{j}$ is the $j$-th row of the coefficient matrix $\mathrm{A} \in \mathbb{F}_{\mathrm{q}}^{J \times K}$. Formally, a $\left(2^{n R_{1}}, \ldots, 2^{n R_{K}}, n\right)$ distributed lossy computation code consists of

- $K$ encoders, where encoder $k \in \mathcal{K}$ assigns an index $m_{k}\left(x_{k}^{n}\right) \in\left[2^{n R_{k}}\right]$ to each sequence $x_{k}^{n} \in \mathbb{F}_{\mathrm{q}}^{n}$;

- a decoder that assigns $J$ estimates $\hat{w}_{\mathrm{A}_{j}}^{n} \in \mathbb{F}_{\mathrm{q}}^{n}, j \in[1: J]$ to each index tuple $\left(m_{1}, \ldots, m_{K}\right) \in\left[2^{n R_{1}}\right] \times \cdots \times\left[2^{n R_{K}}\right]$ and side information $y^{n} \in \mathcal{Y}^{n}$.

A rate tuple $\left(R_{1}, \ldots, R_{K}\right)$ is said to be achievable for Acomputation with distortion tuple $\left(D_{1}, \ldots, D_{J}\right)$ if there exists a sequence of $\left(2^{n R_{1}}, \ldots, 2^{n R_{K}}, n\right)$ codes that satisfy

$$
\limsup _{n \rightarrow \infty} \frac{1}{n} \sum_{i=1}^{n} \mathrm{E}\left(d_{j}\left(W_{\mathrm{A}_{j}, i}, \hat{W}_{\mathrm{A}_{j}, i}\right)\right) \leq D_{j}, \quad j \in[1: J] .
$$

The rate-distortion region $\mathscr{R}^{*}\left(D_{1}, \ldots, D_{J}\right)$ for distributed lossy computation is the closure of the set of all achievable rate tuples $\left(R_{1}, \ldots, R_{K}\right)$ for distortion $\left(D_{1}, \ldots, D_{J}\right)$.

Remark 1: The problem statement specializes to the Körner-Marton (KM) problem [1] if $K=2, J=1, \mathrm{q}=2$, $\mathrm{A}=[1,1], d_{1}, d_{2}$ are Hamming distortion measures, $Y=\emptyset$, and $D_{1}=D_{2}=0$.

Remark 2: The problem statement specializes to the Berger-Tung [17], [18] (BT) distributed lossy source coding problem if $\mathrm{A}$ is the identity matrix and $Y=\emptyset$.

\section{MAIN RESULTS}

The following theorem establishes an achievable rate region for A-computation using nested linear codes (NLC).

Theorem 1 (Lossy computation of correlated sources):

A rate tuple $\left(R_{1}, \ldots, R_{K}\right)$ is achievable for recovering the linear combinations with coefficient matrix $\mathrm{A} \in \mathbb{F}_{\mathrm{q}}^{J \times K}$ and distortion tuple $\left(D_{1}, \ldots, D_{J}\right)$ if, for some choice of test channels $\prod_{k=1}^{K} p\left(u_{k} \mid x_{k}\right)$ such that $\hat{W}_{\mathrm{A}}=\mathrm{A}\left[U_{1}, \ldots, U_{K}\right]^{T}$ meets the distortion constraint

$$
\mathrm{E}\left(d_{j}\left(W_{\mathrm{A}_{j}}, \hat{W}_{\mathrm{A}_{j}}\right)\right) \leq D_{j}, \quad j \in[1: J],
$$

it is contained in

$$
\begin{aligned}
\mathscr{R}= & \bigcup_{\mathrm{B}} \bigcap_{\mathrm{C}} \bigcup_{\mathcal{S}} \bigcap_{\mathcal{T}}\left\{\left(R_{1}, \ldots, R_{K}\right) \in \mathbb{R}_{+}^{K}:\right. \\
& \left.\sum_{k \in \mathcal{T}} R_{k}>H\left(\hat{W}_{\mathrm{B}} \mid Y, \hat{W}_{\mathrm{CB}}\right)-H(U(\mathcal{T}) \mid X(\mathcal{T}))\right\}
\end{aligned}
$$

where $\hat{W}_{\mathrm{B}}=\mathrm{B}\left[U_{1}, \ldots, U_{K}\right]^{T}, \hat{W}_{\mathrm{CB}}=\mathrm{CB}\left[U_{1}, \ldots, U_{K}\right]^{T}$, and the set operations are over all tuples $(B, C, \mathcal{S}, \mathcal{T})$ satisfying the following constraints:

1) $\mathrm{B} \in \mathbb{F}_{\mathrm{q}}^{L_{\mathrm{B}} \times K}$ runs over all full-rank matrices such that $1 \leq L_{\mathrm{B}} \leq K$ and $\operatorname{span}(\mathrm{B}) \supseteq \operatorname{span}(\mathrm{A})$

2) $\mathrm{C} \in \mathbb{F}_{\mathrm{q}}^{L_{\mathrm{C}} \times L_{\mathrm{B}}}$ runs over all full-rank matrices (including empty matrices) such that $0 \leq L_{\mathrm{C}}<L_{\mathrm{B}}$,

3) $\mathcal{S} \subseteq\left[1: L_{\mathrm{B}}\right]$ runs over all index sets of size $|\mathcal{S}|=L_{\mathrm{B}}-L_{\mathrm{C}}$ satisfying

$$
\operatorname{rank}\left(\left[\begin{array}{c}
\mathrm{C} \\
\mathbf{I}_{L_{\mathrm{B}}}(\mathcal{S})
\end{array}\right]\right)=L_{\mathrm{B}}
$$

4) $\mathcal{T} \subseteq \mathcal{K}$ runs over all index sets of size $|\mathcal{T}|=L_{\mathrm{B}}-L_{\mathrm{C}}$ satisfying

$$
\operatorname{rank}\left(\left[\begin{array}{c}
\mathrm{B}(\mathcal{S}) \\
\mathbf{I}_{K}(\mathcal{K} \backslash \mathcal{T})
\end{array}\right]\right)=K .
$$

To build up intuition about this rate region, we now explore several two-user special cases. We begin with the Berger-Tung problem [18], which corresponds to $K=J=2, Y=\emptyset$, and $\mathrm{A}=\mathbf{I}$

Corollary 1 (Two-user distributed source coding via NLC): Consider the setting where $K=J=2, Y=\emptyset$, and $\mathrm{A}=\mathbf{I}$. Then, the set of inequalities (2) in Theorem 1 simplify to $\mathscr{R}_{\mathrm{LDSC}}\left(D_{1}, D_{2}\right):=\mathscr{R}_{\mathrm{BT}}\left(D_{1}, D_{2}\right) \cap \mathscr{R}_{1}\left(D_{1}, D_{2}\right)$ where $\mathscr{R}_{\mathrm{BT}}\left(D_{1}, D_{2}\right)$ is the set of rate pairs that satisfy

$$
\begin{aligned}
R_{1}>I\left(U_{1} ; X_{1} \mid U_{2}\right), \\
R_{2}>I\left(U_{2} ; X_{2} \mid U_{1}\right), \\
R_{1}+R_{2}>I\left(U_{1}, U_{2} ; X_{1}, X_{2}\right),
\end{aligned}
$$

and $\mathscr{R}_{1}\left(D_{1}, D_{2}\right)$ is the set of rate pairs that satisfy

$$
\begin{aligned}
& R_{1}>\max _{\mathrm{B}}\left\{I\left(U_{1} ; X_{1}\right)-I\left(U_{1} ; \hat{W}_{\mathrm{B}}\right)\right\}, \\
& \text { or } \\
& R_{2}>\max _{\mathrm{B}}\left\{I\left(U_{2} ; X_{2}\right)-I\left(U_{2} ; \hat{W}_{\mathrm{B}}\right)\right\},
\end{aligned}
$$

$\hat{W}_{\mathrm{B}}=\mathrm{B} U_{\mathcal{K}}^{\top}$, and the maximization is over all $\mathrm{B} \in \mathbb{F}_{\mathrm{q}}^{1 \times 2}$ such that there is no zero element in B. 
Remark 3: Note that $\mathscr{R}_{\mathrm{BT}}$ is the Berger-Tung region attainable via random i.i.d. codes (see, e.g., [15, Thm. 12.1]). Therefore, for any choice of auxiliary random variables $U_{1}, U_{2}$, the nested linear coding region is contained within the i.i.d. coding region, $\mathscr{R}_{\mathrm{LDSC}}\left(D_{1}, D_{2}\right) \subset \mathscr{R}_{\mathrm{BT}}\left(D_{1}, D_{2}\right)$. A similar phenomenon occurs for the nested linear coding region for the multiple-access channel. However, recent work by Sen and Kim [12] has shown that, for any rate pair in the multipleaccess capacity region, one can always select a finite field and pmf such that this rate pair is included in the nested linear coding region. An interesting question for future study is whether the same is true for distributed source coding.

We now consider the scenario where we wish to recover a single linear combination of two sources.

Definition 1: For $K=2$ and $J=1$, let $\mathscr{R}_{\text {Comp }}(D)$ be the set of rate pairs $\left(R_{1}, R_{2}\right)$ such that $\left(R_{1}, R_{2}\right) \in \mathscr{R}_{\text {Comp }}(D)$ if there exists some $p\left(u_{1} \mid x_{1}\right) p\left(u_{2} \mid x_{2}\right), \hat{w}_{\mathrm{A}}=\mathrm{A}\left[u_{1}, u_{2}\right]^{\top}$ such that $\mathrm{E}\left(d_{1}\left(W_{\mathrm{A}}, \hat{W}_{\mathrm{A}}\right)\right) \leq D$, and it satisfies

$$
\begin{aligned}
& R_{1}>H\left(\hat{W}_{\mathrm{A}} \mid Y\right)-H\left(U_{1} \mid X_{1}\right), \\
& R_{2}>H\left(\hat{W}_{\mathrm{A}} \mid Y\right)-H\left(U_{2} \mid X_{2}\right) .
\end{aligned}
$$

It is sometimes useful to focus on the coefficient vector $A \in$ $\mathbb{F}_{\mathrm{q}}^{1 \times 2}$ that simultaneously minimizes both constraints in (5). We refer to this choice as the "natural computation."

Theorem 2 (Two users, one linear combination): For $K=$ 2 and $J=1$, a rate pair $\left(R_{1}, R_{2}\right)$ is achievable for Acomputation with distortion $D$ if $\left(R_{1}, R_{2}\right) \in \mathscr{R}_{\text {Comp }}(D) \cup$ $\mathscr{R}_{\text {LDSC }}\left(D_{1}, D_{2}\right)$ for some $p\left(u_{1} \mid x_{1}\right) p\left(u_{2} \mid x_{2}\right), \hat{w}_{\mathrm{A}}=\mathrm{A}\left[u_{1}, u_{2}\right]^{\top}$ such that $\mathrm{E}\left(d_{1}\left(W_{\mathrm{A}}, \hat{W}_{\mathrm{A}}\right)\right) \leq D$. (The distortions $D_{1}, D_{2}$ may take any values so long as the distortion constraint for $W_{\mathrm{A}}$ is met.)

We now comment on the two component regions, $\mathscr{R}_{\text {Comp }}(D)$ and $\mathscr{R}_{\text {LDSC }}\left(D_{1}, D_{2}\right)$. The rate region $\mathscr{R}_{\text {Comp }}(D)$ can be viewed as a generalization of the Körner-Marton [1] lossless computation rate region to the lossy case. As discussed in Remark 3, the distributed source coding rate region $\mathscr{R}_{\mathrm{LDSC}}\left(D_{1}, D_{2}\right)$ is included in the Berger-Tung rate region for any choice of test channels. On the contrary, if $A$ is taken to be the natural computation, then, for any choice of test channels, the union $\mathscr{R}_{\text {Comp }}(D) \cup \mathscr{R}_{\text {LDSC }}\left(D_{1}, D_{2}\right)$ includes the Berger-Tung rate region. To illustrate this effect, consider the lossless case, where this rate region simplifies to the union of the SlepianWolf rate region and the Körner-Marton rate region. As shown in Figure 2, there is a defect in $\mathscr{R}_{\mathrm{LDSC}}$ that is filled in by $\mathscr{R}_{\mathrm{KM}}$.

Remark 4: Note that the union rate region results from simultaneous joint typicality decoding and is attained without coded time sharing.

We now consider lossless examples of the special cases explored above.

Example 1 (Two-user lossless source coding via NLC): Consider the lossless case of the setting in Corollary 1. Then, the set of inequalities (2) simplify to $\mathscr{R}_{\mathrm{SW}} \cap \mathscr{R}_{2}$ where $\mathscr{R}_{\mathrm{SW}}$ is the set of rate pairs that satisfy

$$
\begin{aligned}
& R_{1}>H\left(X_{1} \mid X_{2}\right), \\
& R_{2}>H\left(X_{2} \mid X_{1}\right),
\end{aligned}
$$

$$
R_{1}+R_{2}>H\left(X_{1}, X_{2}\right),
$$

and $\mathscr{R}_{2}$ is the set of rate pairs that satisfy

$$
R_{1}>\max _{\mathrm{B}} H\left(X_{1} \mid W_{\mathrm{B}}\right), \text { or } R_{2}>\max _{\mathrm{B}} H\left(X_{2} \mid W_{\mathrm{B}}\right),
$$

where the maximum is over all $B \in \mathbb{F}_{\mathrm{q}}^{1 \times 2}$ such that there is no zero element in $\mathrm{B}$.

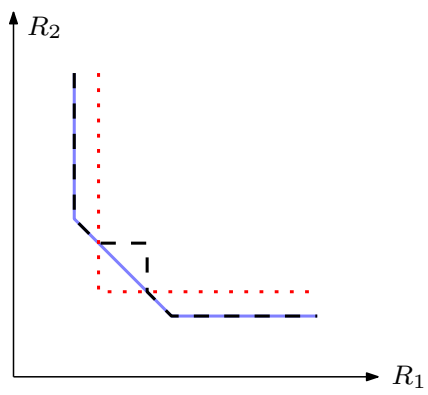

Fig. 2. Example rate region for the lossless two-user one linear computation case. The solid line region is the Slepian-Wolf rate region, the dotted line region is $\mathscr{R}_{\mathrm{KM}}$, and the dashed line region is $\mathscr{R}_{\mathrm{LDSC}}$. Note that the union $\mathscr{R}_{\mathrm{KM}} \cup \mathscr{R}_{\mathrm{LDSC}}$ includes the Slepian-Wolf rate region. Moreover, if the sources are doubly symmetric binary sources $\mathscr{R}_{\mathrm{KM}}$ is optimal.

Example 2 (Körner-Marton [1]): Consider the lossless case with $K=2, J=1, Y=\emptyset$. Then, by choosing $U_{k}=X_{k}, k=1,2$, the set of inequalities in Theorem 1 simplify to $\mathscr{R}_{\mathrm{SW}} \cup \mathscr{R}_{\mathrm{KM}}$ where $\mathscr{R}_{\mathrm{KM}}$ is the set of rate pairs such that

$$
R_{1}>H\left(W_{\mathrm{A}}\right), \quad R_{2}>H\left(W_{\mathrm{A}}\right) .
$$

The rate region $\mathscr{R}_{\mathrm{KM}}$ is the rate region of Körner-Marton [1]. In general, with a notable exception to doubly symmetric sources, $\mathscr{R}_{\mathrm{KM}}$ is suboptimal since neither $\mathscr{R}_{\mathrm{SW}}$ or $\mathscr{R}_{\mathrm{KM}}$ includes the other (and the Slepian-Wolf rate region is also an achievable rate region for computation). This example demonstrates that, with simultaneous joint typicality decoding, the rate region can be improved by including the Slepian-Wolf rate region $\mathscr{R}_{\mathrm{SW}}$ without the need for time sharing.

\section{Sketch of the Proof of Theorem 1}

In this section, we present our linear coding strategy and proof of our main theorem. We first specify the nested linear codes that will be used as our encoding functions in this paper. User $k \in \mathcal{K}$ uses two indices $m_{k} \in\left[2^{n R_{k}}\right]$ and $l_{k} \in\left[2^{n \hat{R}_{k}}\right]$ with rates $R_{k}$ and $\hat{R}_{k}$, respectively. We define $R_{\max }:=$ $\max \left\{R_{1}, \ldots, R_{K}\right\}$, and $\hat{R}_{\max }:=\max \left\{\hat{R}_{1}, \ldots, \hat{R}_{K}\right\}$.

Definition 2 (Expansion of indices in $\mathbb{F}_{\mathrm{q}}$ ): Let us define $\mathrm{m}_{k}\left(m_{k}\right), m_{k} \in\left[2^{n R_{k}}\right]$ as the q-ary expansions of $m_{k}$, which we zero-pad to length $\left\lceil n R_{\max } / \log (\mathrm{q})\right\rceil$ whenever $R_{k}<R_{\max }$. Similarly, we define $l_{k}\left(l_{k}\right), l_{k} \in\left[2^{n \hat{R}_{k}}\right]$ as the q-ary expansions of $l_{k}$, zero-padded to length $\left\lceil n \hat{R}_{\max } / \log (\mathbf{q})\right\rceil$ whenever $\hat{R}_{k}<\hat{R}_{\max }$. For simplicity, we assume that $n R_{k} / \log (\mathbf{q})$ and $n \hat{R}_{k} / \log (\mathrm{q})$ are integers for all rates in the sequel. Since $\mathrm{m}_{k}$ and $m_{k}\left(l_{k}\right.$ and $\left.l_{k}\right)$ are one-to-one mapped, we will use them interchangeably throughout. For example, $\mathrm{m}_{k} \in\left[2^{n R_{k}}\right]$ will be used to denote $\mathrm{m}_{k}\left(m_{k}\right), m_{k} \in\left[2^{n R_{k}}\right]$. 
We define a $\left(2^{n R_{1}}, \ldots, 2^{n R_{K}}, 2^{n \hat{R}_{1}}, \ldots, 2^{n \hat{R}_{K}}, \mathrm{q}, n\right)$ nested linear code as the collection of $K$ codebooks generated by the following procedure.

Fix a distribution $\prod_{k=1}^{K} p\left(u_{k} \mid x_{k}\right)$ and reconstruction functions $\hat{w}_{\mathrm{A}_{j}}=\mathrm{A}_{j}\left[u_{1}, \ldots, u_{K}\right]^{\top}$ such that $\mathrm{E}\left[d_{j}\left(W_{\mathrm{A}_{j}}, \hat{W}_{\mathrm{A}_{j}}\right)\right] \leq$ $D_{j} /\left(1+\epsilon^{\prime \prime}\right), \quad j \in[1: J]$ and assume $\epsilon^{\prime \prime}>\epsilon^{\prime}>\epsilon$.

Codebook generation. Fix a finite field $\mathbb{F}_{\mathrm{q}}$ and a parameter $\epsilon^{\prime} \in(0,1)$. Randomly generate a $\kappa \times n$ matrix, $\mathrm{G} \in \mathbb{F}_{\mathrm{q}}^{\kappa \times n}$, and dithers $\mathrm{d}_{k}^{n} \in \mathbb{F}_{\mathrm{q}}^{n}, k=1, \ldots, K$ where $\kappa=n\left(R_{\max }+\right.$ $\left.\hat{R}_{\text {max }}\right) / \log (\mathrm{q})$ and each element of $\mathrm{G}$ and $\mathrm{d}_{k}^{n}$ are independently and randomly generated according to $\operatorname{Unif}\left(\mathbb{F}_{\mathrm{q}}\right)$.

For each $k \in \mathcal{K}$, generate a linear code $\mathcal{C}_{k}$ by

$$
\begin{aligned}
u_{k}^{n}\left(m_{k}, l_{k}\right) & :=u_{k}^{n}\left(\mathrm{~m}_{k}, \mathrm{l}_{k}\right) \\
& =\left[\mathrm{m}_{k}, \mathrm{I}_{k}\right] \mathrm{G} \oplus \mathrm{d}_{k}^{n},
\end{aligned}
$$

for $m_{k} \in\left[2^{n R_{k}}\right], l_{k} \in\left[2^{n \hat{R}_{k}}\right]$. Note that from this construction, each codeword is i.i.d. uniformly distributed (i.e., $\prod_{i=1}^{n} p_{\mathrm{q}}\left(u_{k i}\right)$ where $\left.p_{\mathrm{q}}=\operatorname{Unif}\left(\mathbb{F}_{\mathrm{q}}\right)\right)$, and the codewords are pairwise independent.

Encoding. For $k \in \mathcal{K}$, find an index pair $\left(m_{k}, l_{k}\right) \in\left[2^{n R_{k}}\right] \times$ $\left[2^{n \hat{R}_{k}}\right]$ such that

$$
\left(u_{k}^{n}\left(m_{k}, l_{k}\right), x_{k}^{n}\right) \in \mathcal{T}_{\epsilon^{\prime}}^{(n)}\left(U_{k}, X_{k}\right) .
$$

If there is more than one such index pair, select one randomly and uniformly. If there is none, randomly choose an index pair from $\left[2^{n R_{k}}\right] \times\left[2^{n \hat{R}_{k}}\right]$. Node $k$ sends $m_{k}$.

Definition 3 (Linear combination of indices): For a given coefficient matrix $\mathrm{A} \in \mathbb{F}_{\mathrm{q}}^{J \times K}$, we define linear combinations

$\mathrm{m}_{\mathrm{A}}\left(\mathrm{m}_{1}, \ldots, \mathrm{m}_{K}\right)=\mathrm{A}\left[\begin{array}{c}\mathrm{m}_{1} \\ \vdots \\ \mathrm{m}_{K}\end{array}\right], \mathrm{I}_{\mathrm{A}}\left(\mathrm{I}_{1}, \ldots, \mathrm{l}_{K}\right)=\mathrm{A}\left[\begin{array}{c}\mathrm{I}_{1} \\ \vdots \\ \mathrm{I}_{K}\end{array}\right]$.

When it is clear from the context, we will simply use the short hand $\mathrm{m}_{\mathrm{A}}$ and $\mathrm{I}_{\mathrm{A}}$ in place of $\mathrm{m}_{\mathrm{A}}\left(\mathrm{m}_{1}, \ldots, \mathrm{m}_{K}\right)$ and $\mathrm{I}_{\mathrm{A}}\left(\mathrm{I}_{1}, \ldots, \mathrm{I}_{K}\right)$, respectively. Note that the above definition implies $\mathrm{m}_{\mathrm{A}_{j}}=\bigoplus_{k=1}^{K} \mathrm{~A}_{j k} \mathrm{~m}_{k}$ and $\mathrm{I}_{\mathrm{A}_{j}}=\bigoplus_{k=1}^{K} \mathrm{~A}_{j k} \mathrm{I}_{k}$, where $\mathrm{A}_{j}$ is the $j$-th row of $\mathrm{A}_{j}$.

Decoding. Let $\epsilon^{\prime}<\epsilon$. Upon receiving $\left(\mathrm{m}_{1}, \ldots, \mathrm{m}_{K}\right)$ and observing $y^{n}$, the decoder searches for a unique index tuple $\hat{\mathrm{I}}_{\mathrm{A}}\left(\hat{\mathrm{I}}_{1}, \ldots, \hat{\mathrm{I}}_{K}\right)$ such that

$$
\left(u_{1}^{n}\left(\mathrm{~m}_{1}, \hat{\mathrm{l}}_{1}\right), \ldots, u_{K}^{n}\left(\mathrm{~m}_{K}, \hat{\mathrm{I}}_{K}\right), y^{n}\right) \in \mathcal{T}_{\epsilon}^{(n)},
$$

for some $\hat{\mathrm{I}}_{k} \in\left[2^{n \hat{R}_{k}}\right], k \in \mathcal{K}$. If it finds a unique index tuple, it declares

$$
\hat{w}_{\mathrm{A}}^{n}\left(\mathrm{~m}_{\mathrm{A}}, \hat{\mathrm{I}}_{\mathrm{A}}\right)=\left[\mathrm{m}_{\mathrm{A}}, \hat{\mathrm{l}}_{\mathrm{A}}\right] \mathrm{G} \oplus \mathrm{d}_{\mathrm{A}}^{n}
$$

as its estimate where $m_{A}=m_{A}\left(m_{1}, \ldots, m_{K}\right)$. Otherwise, if there is no such index tuple, or more than one, the decoder declares an error.

Analysis of the expected distortion. Let $\left(\mathrm{M}_{k}, \mathrm{~L}_{k}\right), k \in \mathcal{K}$ be the indices chosen by the encoders and let $\hat{L}_{\mathrm{A}}, k \in \mathcal{K}$ be the decoded indices. Define the "error" event

$$
\mathcal{E}=\left\{\left(X_{1}^{n}, \ldots, X_{K}^{n}, W_{\mathrm{A}}^{n}\left(\mathrm{M}_{\mathrm{A}}, \hat{\mathrm{L}}_{\mathrm{A}}\right), Y^{n}\right) \notin \mathcal{T}_{\epsilon}^{(n)}\right\} .
$$

By the typical average lemma [15], if the probability of "error" $\mathrm{P}(\mathcal{E})$ tends to zero as $n \rightarrow \infty$ the average distortions over the random codebooks are bounded as desired. Thus, in the remainder of this section, we show that $\mathrm{P}(\mathcal{E})$ tends to zero as $n \rightarrow \infty$ if the conditions in Theorem 1 are satisfied.

First, we divide $\mathcal{E}$ into the events

$$
\begin{aligned}
\mathcal{E}_{1}=\{ & \left(U_{k}^{n}\left(m_{k}, l_{k}\right), X_{k}^{n}\right) \notin \mathcal{T}_{\epsilon^{\prime}}^{(n)} \text { for all }\left(m_{k}, l_{k}\right) \\
& \text { for some } k \in \mathcal{K}\}, \\
\mathcal{E}_{2}=\{ & \left.\left(X_{\mathcal{K}}^{n}, U_{1}^{n}\left(\mathrm{M}_{1}, \mathrm{~L}_{1}\right), \ldots, U_{K}^{n}\left(\mathrm{M}_{K}, \mathrm{~L}_{K}\right), Y^{n}\right) \notin \mathcal{T}_{\epsilon}^{(n)}\right\} \\
\mathcal{E}_{3}=\{ & \left(U_{1}^{n}\left(\mathrm{M}_{1}, \hat{\mathrm{I}}_{1}\right), \ldots, U_{K}^{n}\left(\mathrm{M}_{K}, \hat{\mathrm{I}}_{K}\right), Y^{n}\right) \in \mathcal{T}_{\epsilon}^{(n)} \text { for some } \\
& \left.\left.\left(\hat{\mathrm{I}}_{1}, \ldots, \hat{\mathrm{I}}_{K}\right) \text { such that } \hat{\mathrm{I}}_{\mathrm{A}}\left(\hat{\mathrm{I}}_{1}, \ldots, \hat{\mathrm{I}}_{K}\right) \neq \mathrm{L}_{\mathrm{A}}\right)\right\} .
\end{aligned}
$$

Since $\mathcal{E}=\mathcal{E}_{1} \cup \mathcal{E}_{2} \cup \mathcal{E}_{3}$, by the union of events bound,

$$
\mathrm{P}(\mathcal{E}) \leq \mathrm{P}\left(\mathcal{E}_{1}\right)+\mathrm{P}\left(\mathcal{E}_{2} \cap \mathcal{E}_{1}^{c}\right)+\mathrm{P}\left(\mathcal{E}_{3} \cap \mathcal{E}_{1}^{c}\right) .
$$

By the covering lemma in [11, Lemma 9], the probability $\mathrm{P}\left(\mathcal{E}_{1}\right)$ tends to zero as $n \rightarrow \infty$ if

$$
R_{k}+\hat{R}_{k}>I\left(U_{k} ; X_{k}\right)+D\left(p_{U_{k}} \| p_{\mathbf{q}}\right)+\delta\left(\epsilon^{\prime}\right), \quad k \in \mathcal{K} .
$$

Define $\mathcal{M}:=\left\{\mathrm{M}_{1}=\mathbf{0}, \ldots, \mathrm{M}_{K}=\mathbf{0}, \mathrm{L}_{1}=\mathbf{0}, \ldots, \mathrm{L}_{K}=\right.$ 0 $\}$ as the event where all the chosen indices are zero. By symmetry of the codebook construction and encoding steps, we have that $P\left(\mathcal{E}_{2} \cap \mathcal{E}_{1}^{c}\right)=P\left(\mathcal{E}_{2} \cap \mathcal{E}_{1}^{c} \mid \mathcal{M}\right)$ and $P\left(\mathcal{E}_{3} \cap \mathcal{E}_{1}^{c}\right)=$ $P\left(\mathcal{E}_{3} \cap \mathcal{E}_{1}^{c} \mid \mathcal{M}\right)$. By the Markov lemma in [11, Lemma 12], the second term $\mathrm{P}\left(\mathcal{E}_{2} \cap \mathcal{E}_{1}^{c} \mid \mathcal{M}\right)$ tends to zero as $n \rightarrow \infty$ if (11) is satisfied. In the following, we use the shorthand notation $U_{k}^{n}\left(\hat{\mathrm{I}}_{k}\right):=U_{k}^{n}\left(\mathbf{0}, \hat{l}_{k}\right)$ for simplicity.

Let $\mathrm{B} \in \mathbb{F}_{\mathrm{q}}^{J_{\mathrm{B}} \times K}$ be any rank $J_{\mathrm{B}}$ matrix $\left(L \leq J_{\mathrm{B}} \leq K\right)$ such that $\operatorname{span}(A) \subseteq \operatorname{span}(B)$. Then, the third probability term in (10) can be bounded by,

$$
\begin{aligned}
& \mathrm{P}\left(\mathcal{E}_{3} \cap \mathcal{E}_{1}^{c} \mid \mathcal{M}\right) \\
& =\mathrm{P}\left\{\left(U_{1}^{n}\left(\hat{\mathrm{I}}_{1}\right), \ldots, U_{K}^{n}\left(\hat{\mathrm{I}}_{K}\right), Y^{n}\right) \in \mathcal{T}_{\epsilon}^{(n)}\right. \text { for some } \\
& \left.\left(\hat{\mathrm{I}}_{1}, \ldots, \hat{\mathrm{I}}_{K}\right) \text { such that } \hat{\mathrm{I}}_{\mathrm{A}} \neq \mathbf{0}, \mathcal{E}_{1}^{c} \mid \mathcal{M}\right\} \\
& \stackrel{(a)}{=} \mathrm{P}\left\{\left(U_{1}^{n}\left(\hat{\mathrm{I}}_{1}\right), \ldots, U_{K}^{n}\left(\hat{\mathrm{I}}_{K}\right), \hat{W}_{\mathrm{B}}^{n}\left(\hat{\mathrm{I}}_{\mathrm{B}}\right), Y^{n}\right) \in \mathcal{T}_{\epsilon}^{(n)}\right. \text { for some } \\
& \left.\left(\hat{\imath}_{1}, \ldots, \hat{\imath}_{K}\right) \text { such that } \hat{\imath}_{\mathrm{A}} \neq \mathbf{0}, \mathcal{E}_{1}^{c} \mid \mathcal{M}\right\} \\
& \leq \mathrm{P}\left\{\left(\hat{W}_{\mathrm{B}}^{n}\left(\hat{\mathrm{I}}_{\mathrm{B}}\right), Y^{n}\right) \in \mathcal{T}_{\epsilon}^{(n)} \text { for some }\left(\hat{\mathrm{I}}_{1}, \ldots, \hat{\mathrm{I}}_{K}\right)\right. \\
& \text { such that } \left.\hat{I}_{\mathrm{A}} \neq \mathbf{0}, \mathcal{E}_{1}^{c} \mid \mathcal{M}\right\} \\
& \stackrel{(b)}{\leq} \mathrm{P}\left\{\left(\hat{W}_{\mathrm{B}}^{n}\left(\hat{\mathrm{I}}_{\mathrm{B}}\right), Y^{n}\right) \in \mathcal{T}_{\epsilon}^{(n)} \text { for some }\left(\hat{\mathrm{I}}_{1}, \ldots, \hat{\mathrm{I}}_{K}\right)\right. \\
& \text { such that } \left.\hat{l}_{\mathrm{B}} \neq \mathbf{0}, \mathcal{E}_{1}^{c} \mid \mathcal{M}\right\} \\
& =\mathrm{P}\left\{\left(\hat{W}_{\mathrm{B}}^{n}\left(\hat{\mathrm{l}}_{\mathrm{B}}\right), Y^{n}\right) \in \mathcal{T}_{\epsilon}^{(n)} \text { for some } \hat{\mathrm{l}}_{\mathrm{B}} \neq \mathbf{0}, \mathcal{E}_{1}^{c} \mid \mathcal{M}\right\} \\
& \leq \sum_{\hat{\mathrm{I}}_{\mathrm{B}} \in \mathcal{L}_{\mathrm{B}}} \mathrm{P}\left\{\left(\hat{W}_{\mathrm{B}}^{n}\left(\hat{\mathrm{l}}_{\mathrm{B}}\right), Y^{n}\right) \in \mathcal{T}_{\epsilon}^{(n)}, \mathcal{E}_{1}^{c} \mid \mathcal{M}\right\}
\end{aligned}
$$

where $\hat{\mathrm{I}}_{\mathrm{B}}=\hat{\mathrm{I}}_{\mathrm{B}}\left(\hat{\mathrm{I}}_{1}, \ldots, \hat{\mathrm{I}}_{K}\right), \hat{W}_{\mathrm{B}}^{n}\left(\hat{\mathrm{l}}_{\mathrm{B}}\right)=\left[\mathbf{0}, \hat{\mathrm{I}}_{\mathrm{B}}\right] \mathrm{G} \oplus \mathrm{d}_{\mathrm{B}}^{n}$ and $\mathcal{L}_{\mathrm{B}}=\left\{\hat{\mathrm{I}}_{\mathrm{B}}\left(\hat{\mathrm{I}}_{1}, \ldots, \hat{\mathrm{I}}_{K}\right): \hat{\mathrm{I}}_{\mathrm{B}} \neq \mathbf{0},\left(\hat{\mathrm{I}}_{1}, \ldots, \hat{\mathrm{I}}_{K}\right) \in\left[2^{n \hat{R}_{1}}\right] \times\right.$ $\left.\cdots \times\left[2^{n \hat{R}_{K}}\right]\right\}$. Here, step $(a)$ follows since $\hat{W}_{\mathrm{B}}^{n}\left(\hat{\mathrm{I}}_{\mathrm{B}}\right)$ is a deterministic function of $\left(U_{1}^{n}\left(\hat{l}_{1}\right), \ldots, U_{K}^{n}\left(\hat{l}_{K}\right)\right)$ and step $(b)$ follows since $\hat{l}_{\mathrm{B}}\left(\hat{\mathrm{I}}_{1}, \ldots, \hat{\mathrm{I}}_{K}\right)=\mathbf{0}$ implies $\hat{\mathrm{I}}_{\mathrm{A}}\left(\hat{\mathrm{I}}_{1}, \ldots, \hat{\mathrm{I}}_{K}\right)=\mathbf{0}$. 
Next, we further divide the set $\mathcal{L}_{\mathrm{B}}$ by

$$
\begin{aligned}
\mathcal{L}_{\mathrm{B}}(r, \mathrm{C}) & =\left\{\hat{\mathrm{I}}_{\mathrm{B}}: \hat{\mathrm{I}}_{\mathrm{B}} \in \mathcal{L}_{\mathrm{B}}, \operatorname{rank}\left(\hat{\mathrm{I}}_{\mathrm{B}}\right)=r, \mathrm{C} \hat{\mathrm{B}}_{\mathrm{B}}=\mathbf{0}\right\} \\
& =\left\{\hat{\mathrm{I}}_{\mathrm{B}}: \hat{\mathrm{I}}_{\mathrm{B}} \in \mathcal{L}_{\mathrm{B}}, \operatorname{mullspace}\left(\hat{\mathrm{I}}_{\mathrm{B}}\right)=\operatorname{span}(\mathrm{C})\right\},
\end{aligned}
$$

for $1 \leq r \leq J_{\mathrm{B}}$ and $\mathrm{C} \in \mathbb{F}_{\mathrm{q}}^{\left(J_{\mathrm{B}}-r\right) \times J_{\mathrm{B}}}$. Note that we have $\mathcal{L}_{\mathrm{B}}=\cup_{r} \cup_{\mathrm{C}} \mathcal{L}_{\mathrm{B}}(r, \mathrm{C})$ where $\mathcal{L}_{\mathrm{B}}(r, \mathrm{C})=\mathcal{L}_{\mathrm{B}}\left(r, \mathrm{C}^{\prime}\right)$ if $\operatorname{span}(\mathrm{C})=\operatorname{span}\left(\mathrm{C}^{\prime}\right)$. The set $\mathcal{L}_{\mathrm{B}}(r, \mathrm{C})$ divides $\mathcal{L}_{\mathrm{B}}$ into subsets of $I_{B}$ that have the same left nullspace, where the nullspaces are represented by a generator matrix $C$ (with rank of $J_{\mathrm{B}}-r$ ).

We are now ready to proceed with the last probability term $P\left(\mathcal{E}_{3} \cap \mathcal{E}_{1}^{c} \mid \mathcal{M}\right)$ using the union of events bound. Continuing from (12),

$$
\begin{aligned}
& P\left(\mathcal{E}_{3} \cap \mathcal{E}_{1}^{c} \mid \mathcal{M}\right) \\
& \leq \sum_{\hat{\mathrm{B}}_{\mathrm{B}} \in \mathcal{L}_{\mathrm{B}}} \mathrm{P}\left\{\left(\hat{W}_{\mathrm{B}}^{n}\left(\hat{\mathrm{I}}_{\mathrm{B}}\right), Y^{n}\right) \in \mathcal{T}_{\epsilon}^{(n)}, \mathcal{E}_{1}^{c} \mid \mathcal{M}\right\} \\
& \leq \sum_{r=1}^{J_{\mathrm{B}}} \sum_{\mathrm{C}} \sum_{\hat{\mathrm{I}}_{\mathrm{B}} \in \mathcal{L}_{\mathrm{B}}(r, \mathrm{C})} \mathrm{P}\left\{\left(\hat{W}_{\mathrm{B}}^{n}\left(\hat{\mathrm{I}}_{\mathrm{B}}\right), Y^{n}\right) \in \mathcal{T}_{\epsilon}^{(n)}, \mathcal{E}_{1}^{c} \mid \mathcal{M}\right\}, \\
& \stackrel{(a)}{\leq} \sum_{r=1}^{J_{\mathrm{B}}} \sum_{\mathrm{C}} 2^{n \max _{\mathcal{T}}\left(\sum_{k \in \mathcal{T}} \hat{R}_{k}\right)} \\
& \quad \times 2^{-n\left(I\left(\hat{W}_{\mathrm{B}(\mathcal{S})} ; Y, W_{\mathrm{CB}}\right)+D\left(p_{\hat{W}_{\mathrm{B}(\mathcal{S})}} \| p_{\mathrm{q}}^{|\mathcal{S}|}\right)+\tilde{D}-\delta(\epsilon)\right)},
\end{aligned}
$$

where $\tilde{D}=\sum_{k \in \mathcal{K}}\left[I\left(U_{k} ; X_{k}\right)+D\left(p_{U_{k}} \| p_{\mathrm{q}}\right)-R_{k}-\hat{R}_{k}\right]$ and (a) holds for any $\mathcal{S} \subseteq \mathcal{K}$ such that $|\mathcal{S}|=r$ and

$$
\operatorname{rank}\left(\left[\begin{array}{c}
\mathrm{C} \\
\mathbf{I}_{J_{\mathrm{B}}}(\mathcal{S})
\end{array}\right]\right)=J_{\mathrm{B}},
$$

and the maximum in the exponent of the last inequality is over all $|\mathcal{T}|=r$ such that

$$
\operatorname{rank}\left(\left[\begin{array}{c}
\mathrm{B}(\mathcal{S}) \\
\mathbf{I}_{K}(\mathcal{K} \backslash \mathcal{T})
\end{array}\right]\right)=K .
$$

In step $(a)$ we have applied the following two key lemmas which is a cardinality bound on the set $\mathcal{L}_{\mathrm{B}}(r, \mathrm{C})$ and a joint typicality lemma for nested linear codes. The proof of the lemmas are omitted due to space limitation.

Lemma 1 (Cardinality bound): Let $J_{\mathrm{B}}$ and $r$ be integers such that $J_{\mathrm{B}} \leq K$ and $1 \leq r \leq J_{\mathrm{B}}$. Let $\mathrm{B} \in \mathbb{F}_{\mathrm{q}}^{J_{\mathrm{B}} \times K}, J_{\mathrm{B}} \leq K$ and $\mathrm{C} \in \mathbb{F}_{\mathrm{q}}^{\left(J_{\mathrm{B}}-r\right) \times L_{\mathrm{B}}}$ be full rank matrices. Then, for any $\mathcal{S} \subseteq \mathcal{K}$ such that $|\mathcal{S}|=r$ and $\operatorname{rank}\left(\left[\begin{array}{c}\mathrm{C} \\ \mathbf{I}(\mathcal{S})\end{array}\right]\right)=J_{\mathrm{B}}$, we have that

$$
\left|\mathcal{L}_{\mathrm{B}}(r, \mathrm{C})\right| \leq \max _{\mathcal{T}} 2^{n\left(\sum_{k \in \mathcal{T}} R_{k}+\sum_{k \in \mathcal{T}} \hat{R}_{k}\right)}
$$

where the maximization is over $|\mathcal{T}|=r$ such that

$$
\operatorname{rank}\left(\left[\begin{array}{c}
\mathrm{B}(\mathcal{S}) \\
\mathrm{I}(\mathcal{K} \backslash \mathcal{T})
\end{array}\right]\right)=K .
$$

Lemma 2 (Joint typicality lemma for nested linear codes): Consider $1 \leq r \leq K$ and $\mathrm{C} \in \mathbb{F}_{\mathrm{q}}^{\left(J_{\mathrm{B}}-r\right) \times J_{\mathrm{B}}}$ such that $\operatorname{rank}(\mathrm{C})=J_{\mathrm{B}}-r$ and assume that $\hat{\mathrm{I}}_{\mathrm{B}} \in \mathcal{L}_{\mathrm{B}}(r, \mathrm{C})$. Then,

$$
\begin{aligned}
& \mathrm{P}\left\{\left(\hat{W}_{\mathrm{B}}^{n}\left(\hat{\mathrm{I}}_{\mathrm{B}}\right), Y^{n}\right) \in \mathcal{T}_{\epsilon}^{(n)}, \mathcal{E}_{1}^{c} \mid \mathcal{M}\right\} \\
& \quad \leq 2^{-n\left(I\left(\hat{W}_{\mathrm{B}(\mathcal{S})} ; Y, \hat{W}_{\mathrm{CB}}\right)+D\left(p_{\hat{W}_{\mathrm{B}(\mathcal{S})}} \| p_{\mathrm{q}}^{|\mathcal{S}|}\right)+\tilde{D}-\delta(\epsilon)\right)} .
\end{aligned}
$$

where $\tilde{D}=\sum_{k \in \mathcal{K}}\left[I\left(U_{k} ; X_{k}\right)+D\left(p_{U_{k}} \| p_{\mathrm{q}}\right)-R_{k}-\hat{R}_{k}\right]$.

Thus, for any $\mathrm{B} \in \mathbb{F}_{\mathrm{q}}^{L_{\mathrm{B}} \times K}, \operatorname{span}(\mathrm{A}) \subseteq \operatorname{span}(\mathrm{B})$, we have a bound on $P\left(\mathcal{E}_{3} \cap \mathcal{E}_{1}^{c} \mid \mathcal{M}\right)$ that tends to zero as $n \rightarrow \infty$ if for all full rank $\mathrm{C} \in \mathbb{F}_{\mathrm{q}}^{L_{\mathrm{C}} \times L_{\mathrm{B}}}, 0 \leq L_{\mathrm{C}}<L_{\mathrm{B}}$, there exists an $\mathcal{S}$ that satisfies (13) and

$$
\begin{aligned}
& R_{k}+\hat{R}_{k}>I\left(U_{k} ; X_{k}\right)+D\left(p_{U_{k}} \| p_{\mathrm{q}}\right)+\delta\left(\epsilon^{\prime}\right), \quad k \in \mathcal{K} \\
& \sum_{k \in \mathcal{T}} \hat{R}_{k}<I\left(\hat{W}_{\mathrm{B}(\mathcal{S})} ; Y, \hat{W}_{\mathrm{CB}}\right)+D\left(p_{\hat{W}_{\mathrm{B}(\mathcal{S})}} \| p_{\mathrm{q}}^{|\mathcal{S}|}\right)+\tilde{D}-\delta(\epsilon),
\end{aligned}
$$

for all $\mathcal{T}$ which satisfies (14). The proof is complete by eliminating the auxiliary rates $\hat{R}_{k}, k \in \mathcal{K}$.

\section{REFERENCES}

[1] J. Körner and K. Marton, "How to encode the modulo-two sum of binary sources," IEEE Trans. Inf. Theory, vol. 25, no. 2, pp. 219-221, 1979.

[2] D. Slepian and J. K. Wolf, "Noiseless coding of correlated information sources," IEEE Trans. Inf. Theory, vol. 19, no. 4, pp. 471-480, Jul. 1973.

[3] A. D. Wyner, "Recent results in the Shannon theory," IEEE Trans. Inf. Theory, vol. 20, no. 1, pp. 2-10, 1974.

[4] I. Csiszar, "Linear codes for sources and source networks: Error exponents, universal coding," IEEE Trans. Inf. Theory, vol. 28, no. 4, pp. 585-592, Jul 1982.

[5] T. S. Han and K. Kobayashi, "A dichotomy of functions $F(X, Y)$ of correlated sources $(X, Y)$ from the viewpoint of the achievable rate region," IEEE Trans. Inf. Theory, vol. 33, no. 1, pp. 69-76, 1987.

[6] D. Krithivasan and S. S. Pradhan, "Distributed source coding using Abelian group codes," IEEE Trans. Inf. Theory, vol. 57, no. 3, pp. 14951519, Mar. 2011.

[7] V. Lalitha, N. Prakash, K. Vinodh, P. V. Kumar, and S. S. Pradhan, "Linear coding schemes for the distributed computation of subspaces," IEEE Journal on Selected Areas in Communications, vol. 31, no. 4, pp. 678-690, April 2013.

[8] A. Padakandla, A. G. Sahebi, and S. S. Pradhan, "An achievable rate region for the three-user interference channel based on coset codes," IEEE Trans. Inf. Theory, vol. 62, no. 3, pp. 1250-1279, Mar. 2016.

[9] A. Padakandla and S. S. Pradhan, "An achievable rate region based on coset codes for multiple access channel with states," IEEE Trans. Inf. Theory, vol. 63, no. 10, pp. 6393-6415, Oct. 2017.

[10] — "Achievable rate region for three user discrete broadcast channel based on coset codes," IEEE Trans. Inf. Theory, vol. 64, no. 4, pp. 2267-2297, Apr. 2018.

[11] S. H. Lim, C. Feng, A. Pastore, B. Nazer, and M. Gastpar, "A joint typicality approach to compute-forward," IEEE Trans. Inf. Theory, vol. 64, no. 12, pp. 7657-7685, Dec. 2018.

[12] P. Sen and Y.-H. Kim, "Homologous codes for multiple access channels," Jan. 2018, preprint available at https://arxiv.org/abs/1801.07695.

[13] P. Sen, S. H. Lim, and Y.-H. Kim, "Optimal achievable rates for computation with random homologous codes," May 2018, preprint available at https://arxiv.org/abs/1805.03338.

[14] S. H. Lim, C. Feng, A. Pastore, B. Nazer, and M. Gastpar, "Towards an algebraic network information theory: Simultaneous joint typicality decoding," Jan. 2019, preprint available at https://arxiv.org/abs/1901.03274.

[15] A. El Gamal and Y.-H. Kim, Network Information Theory. Cambridge: Cambridge University Press, 2011.

[16] S. H. Lim, C. Feng, A. Pastore, B. Nazer, and M. Gastpar, "Towards an algebraic network information theory: Simultaneous joint typicality decoding," in Proc. IEEE Int. Symp. Inf. Theory, Aachen, Germany, Jun. 2017.

[17] T. Berger, "Multiterminal source coding," in The Information Theory Approach to Communications, G. Longo, Ed. New York: SpringerVerlag, 1978, pp. 171-231.

[18] S.-Y. Tung, "Multiterminal source coding," Ph.D. Thesis, Cornell University, Ithaca, NY, 1978. 